\title{
Choroid Plexus Papilloma Arising From the Temporal Horn With a Bilateral Hypersecretory Hydrocephalus: A Case Report and Review of Literature
}

\author{
Sureswar Mohanty ${ }^{a}$, Suman Saurav Rout ${ }^{\mathrm{b}, \mathrm{d}}$, Gouri Sankar Sarangi ${ }^{\mathrm{a}}$, Kumudini Devi ${ }^{\mathrm{c}}$
}

\begin{abstract}
Cerebrospinal fluid (CSF) within the cerebral ventricular system is secreted by a neuroepithelial tissue which is called as the choroid plexus. Tumors arising from these tissues are rare. Choroid plexus papillomas (CPPs) have been denoted as WHO grade I of the choroid plexus tumors. Among the intracranial tumors, neoplasms of the choroid plexus constitute around $0.36-0.6 \%$. CPPs are mostly slow growing and cause symptoms due to mass effect and obstructive hydrocephalus, resulting in increased intracranial pressure. We report a case of CPP arising from the temporal horn in a 7-year-old girl presenting with progressive head enlargement since birth due to bilateral massive hydrocephalus without any obstruction, making it purely a hypersecretory hydrocephalus. A drainage procedure followed by complete tumor resection was carried out in our case and the patient showed marked relief from her symptoms.
\end{abstract}

Keywords: Choroid plexus papilloma; Temporal horn

\section{Introduction}

Choroid plexus papillomas (CPPs) are a group of neoplasms that are benign in nature and arise from the choroid plexus which is a structure made from tufts of villi within the ventricular system and secretes cerebrospinal fluid (CSF). CPP rarely involves the third ventricle and is even rarer in the temporal horn. In pediatric age group, almost $80 \%$ of CPPs arise in the lateral ventricle, $16 \%$ involve the fourth ventricle and only $4 \%$

\footnotetext{
Manuscript accepted for publication May 18, 2016
}

aDepartment of Neurosurgery, IMS and SUM Hospital, Siksha 'O' Anusandhan University, Kalinga nagar, Bhubaneswar 751003, Odisha, India

bDepartment of General Surgery, IMS and SUM Hospital, Siksha 'O' Anusandhan University, Kalinga nagar, Bhubaneswar 751003, Odisha, India 'Department of Pathology, IMS and SUM Hospital, Siksha 'O' Anusandhan University, Kalinga nagar, Bhubaneswar 751003, Odisha, India

${ }^{d}$ Corresponding Author: Suman Saurav Rout, Department of General Surgery, IMS and SUM Hospital, Siksha 'O' Anusandhan University, Kalinga nagar, Bhubaneswar 751003, Odisha, India. Email: docssr11@gmail.com occur in the third ventricle. These tumors are benign histologically and are neuroectodermal in origin and assigned a WHO grade I. Complete or gross total resection of these tumors often results in a cure and almost recurrence free survival. The special challenges in the management of these tumors are mostly due to its several unique features which include the young age at presentation, high vascularity of these tumors and the potential for hypersecretion of CSF.

\section{Case Report}

A 7-year-old girl presented with progressive head enlargement since birth, features of raised intracranial pressure in the form of headache, vomiting, excessive crying and excessive drowsiness. Her visual acuity was reduced due to secondary optic atrophy. She had no history of any seizures since birth. Magnetic resonance imaging (MRI) revealed a large hypointense lobulated mass (T2 and FLAIR) originating from the choroid plexus in the temporal horn of the right lateral ventricle measuring almost $50 \times 45 \times 40 \mathrm{~mm}$ in size. All supratentorial ventricles were dilated and the mass was causing compression over adjoining midbrain (Figs. 1-3). With a provisional diagnosis

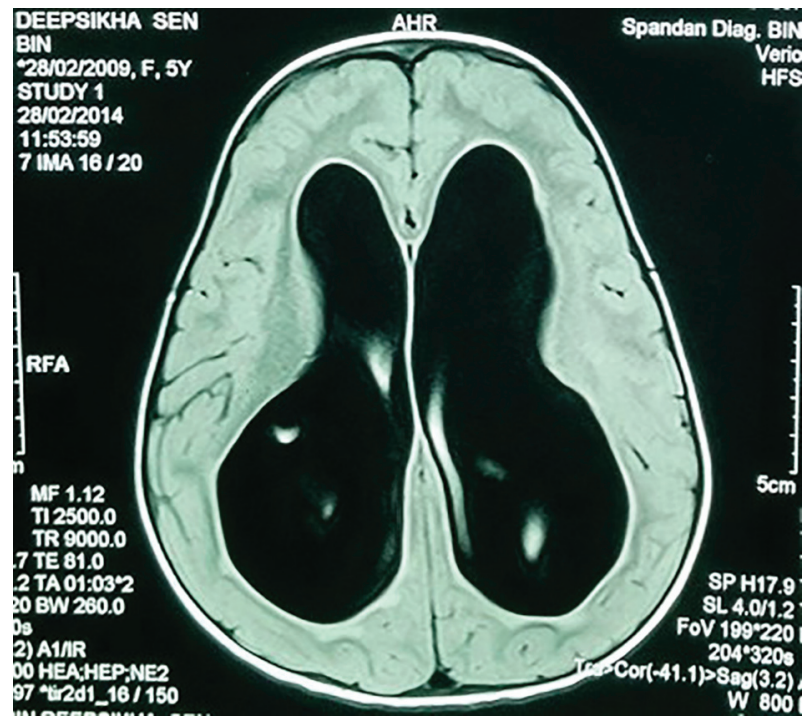

Figure 1. MRI showing large bilateral hydrocephalus. 


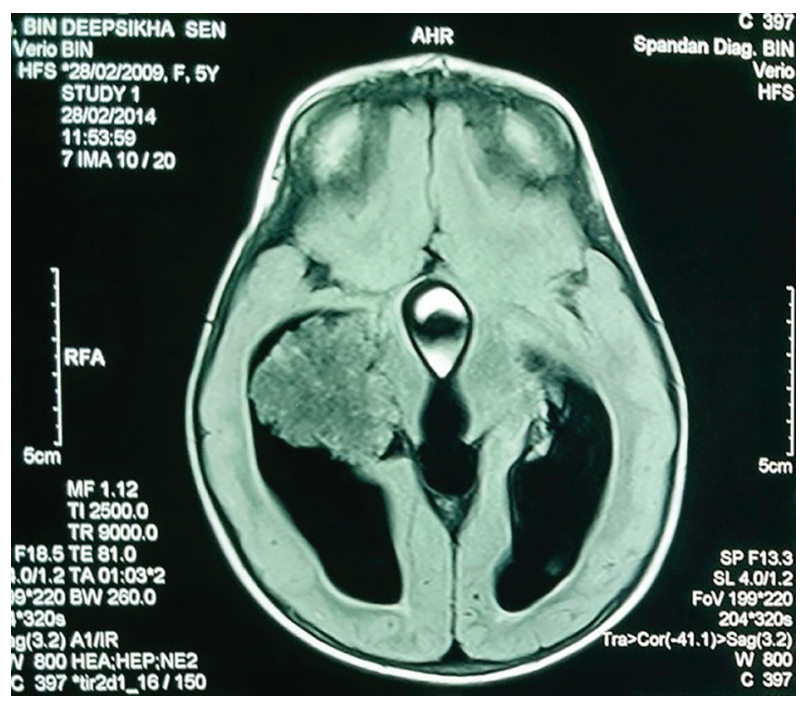

Figure 2. MRI showing the hypointense lobulated mass in the lateral ventricle.

of a choroid plexus mass, the patient was taken up for surgery. A ventriculo-peritoneal shunt was placed for the drainage of excessively forming CSF 2 days prior to the surgery. A right temporal craniotomy was performed and the temporal horn was opened through mid temporal sulcus and the mass was

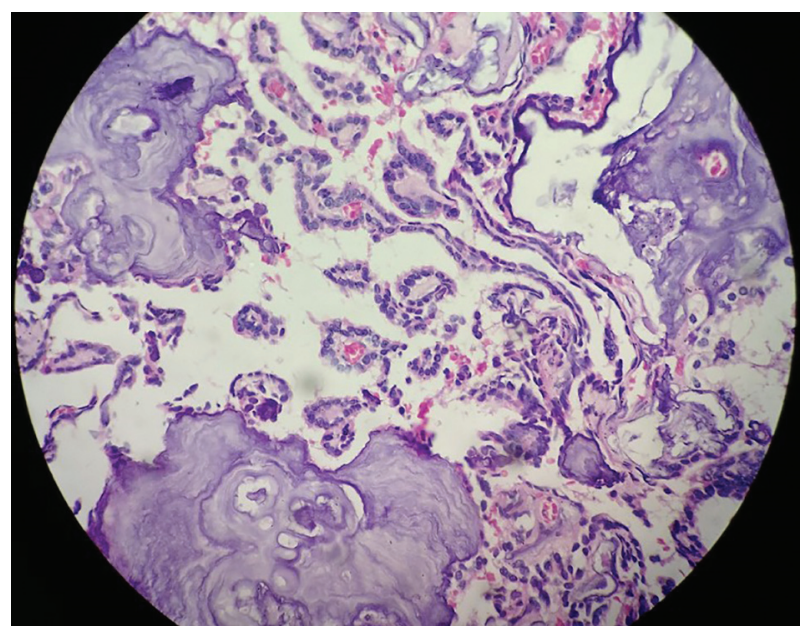

Figure 4. Histopathological image showing fibrovascular fronds lined by cuboidal to low columnar cells with extreme area of calcification and focal areas of chondroid and osseous metaplasia in the stroma.

reached. It was a firm friable and vascular mass arising from the choroid plexus on the temporal horn. The tumor had its vascular supply from the anterior choroidal artery.

Intraoperative squash smear and tissue for frozen section were sent. Cellular cytosmears showing papillary structure with fibrovascular core lined by cuboidal cells having bland

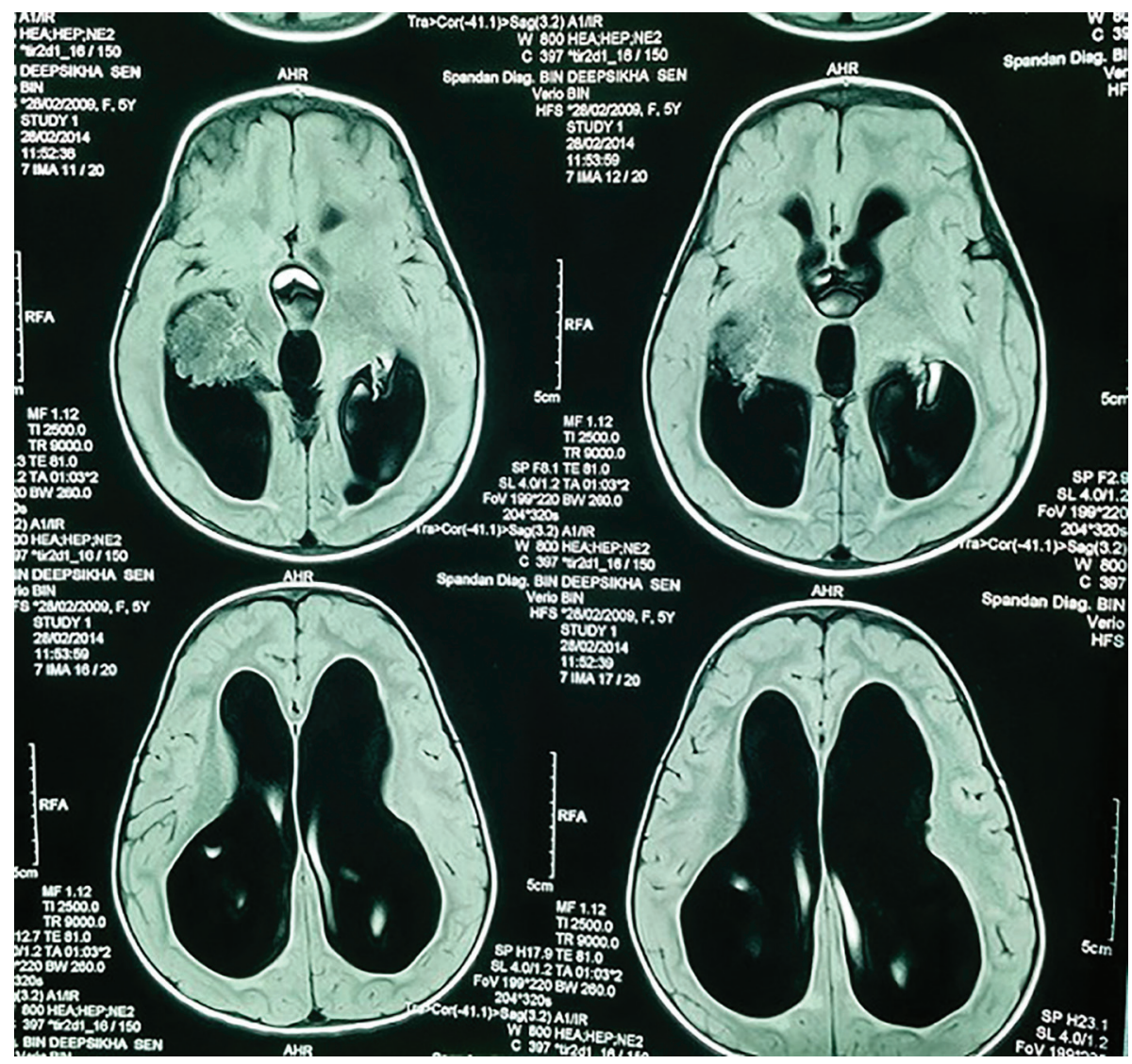

Figure 3. MRI showing lobulated mass and the dilated ventricles. 


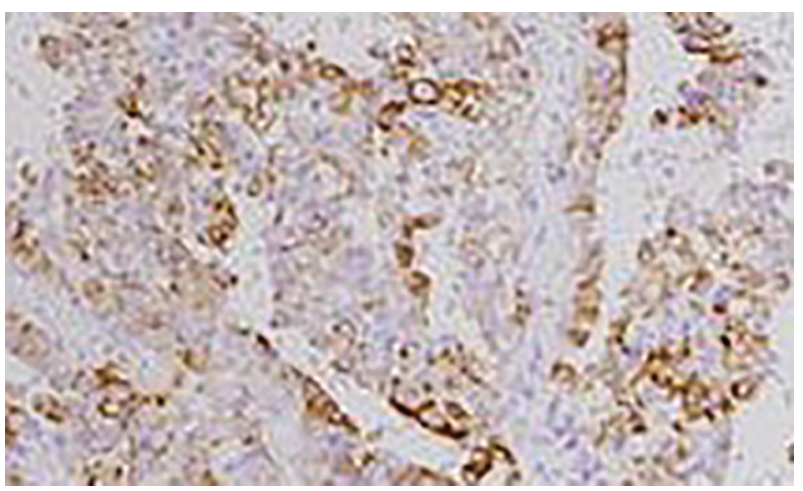

Figure 5. Immunopositivity for cytokeratin (CK7).

nuclei, scanty cytoplasm, present in hemorrhagic background. Flakes of calcification were also present.

Complete removal of the tumor mass was done after securing the vascular supply. There was no element of obstruction. The frozen section and histopathological findings of the excised tumor mass (Microsections showed fibrovascular fronds lined by cuboidal to low columnar cells with extreme area of calcification and focal areas of chondroid and osseous metaplasia in the stroma. No mitosis or necrosis was seen.) were diagnostic of CPP (WHO grade I) (Fig. 4). The immunohistochemistry study showed positivity for cytokeratin (CK7) (Fig. 5) and S-100 (Fig. 6). The patient had an uneventful postoperative period. During the postoperative follow-up after 4 months, the patient was completely symptom free and her visual acuity had improved considerably.

\section{Discussion}

The choroid plexus is the neuroepithelial tissue which produces CSF within the cerebral ventricular system. The atrium of the lateral ventricle rests the largest collection of this tissue. From there, the choroid plexus extends anteriorly towards but not into the temporal horn before entering the foramen of Monro and the third ventricle.

Based on the location of choroidal tissue, the lateral ventricle is the most common site $(50 \%)$ for these tumors, followed by the fourth ventricle $(40 \%)$ and the third ventricle (5\%) [1]. About $5 \%$ of choroid plexus tumors are in more than one location [1]. Apart from these sites there have been sporadic reports of such tumors arising from the extraventricular locations including the suprasellar region, the cerebro-pontine (CP) angle, the frontal lobe, the pineal gland, the posterior commissure, the cerebellum and rarely the temporal horn [29]. On extensive review of literature we could find only few cases of such tumor arising from the temporal horn which presented with either temporal lobe epilepsy or with transtentorial herniation $[10,11]$.

These CPPs have been denoted as WHO grade I of the choroid plexus tumors. Neoplasms of the choroid plexus account for $10-20 \%$ of brain tumors in children younger than 1 year of age, $2-4 \%$ of pediatric brain tumors and $0.36-0.6 \%$ of all intracranial tumors [1]. Almost $20 \%$ of the tumors occur in

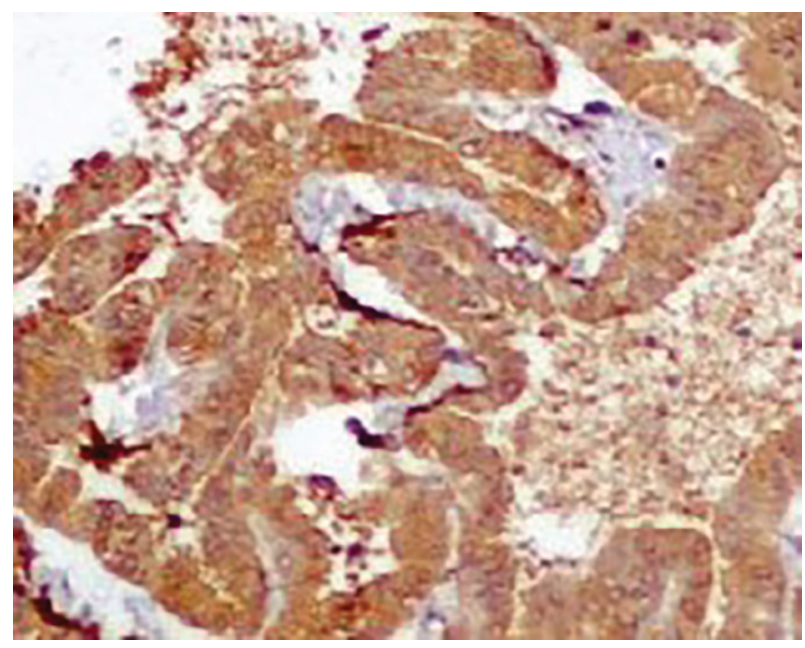

Figure 6. Strong cytoplasmic immunopositivity for S-100.

the first year of life, while $50 \%$ of these tumors manifest in the first decade of life [10].

Choroid plexus tumors occur either as benign slow growing papillomas which have a favorable prognosis overall [1] and constitute about $80 \%$ of them or as biologically highly aggressive carcinomas which make up the other $20 \%$ but are more common in children than in adults. On a whole, these choroid plexus tumors make up a prevalence of around 0.3 per 1 million [1].

There occurs variation in the age of presentation of the tumor which seems to vary with the tumor location. Patients with tumors arising in the lateral ventricle are more frequently in the age group of 10 years or less, while tumors arising in the fourth ventricle tend to occur among patients of age group 0 - 50 years. Males are found to have fourth ventricle tumors more than females, while there is no such definite sex predilection for tumors of the lateral ventricles [1]. In the present case, there was a rapid enlargement of the head soon after birth possibly indicating an intrauterine onset of the tumor.

Most CPPs are slow growing in nature and the symptoms are mostly due to the mass effect and obstructive hydrocephalus along with features of raised ICP. CSF overproduction is a well documented feature of CPPs and the CSF production rate exceeds the normal $450 \mathrm{~mL}$ per day by large amounts [12] and just this exceeding amount irrespective of the presence of obstruction or without it, results in hydrocephalus. Also the fact that postoperative shunts may be required in some patients to treat persistent hydrocephalus and the presence of xanthochromic CSF which is a sign of an ongoing subclinical bleeding supports the theory that impaired CSF absorption at the level of the arachnoid granulations may fall into consideration [12].

Focal neurological deficits, cranial nerve palsies, seizures, coma and psychosis reported in a case [13] are the most common clinical presentations. Li Fraumeni syndrome (p53 mutations) and Aicardi syndrome (X-linked dominant disorder) have been associated with a small number of cases of CPP. Association with human polyoma virus SV40 has been duly noted in some cases.

CPPs are well-circumscribed soft grayish red cauliflower- 
like masses with prominent lobulations peripherally and focal gritty areas due to calcification. Some areas of hemorrhage and cystic degeneration may be seen [1]. The anterior choroidal artery, the lateral posterior choroidal artery, and medial posterior choroidal artery form the main supply of the choroid plexus tumors located in the lateral ventricle [14]. Fourth ventricular choroid plexus tumors are usually supplied by choroidal branches of the posterior inferior cerebellar artery [15]. A vascular pedicle mostly attaches the tumors to the choroid plexus. The trigone region of the choroid plexus is the most common site of attachment of tumors arising in the lateral ventricles, while the tumors of the third ventricle are mostly attached to its roof. In the fourth ventricle, the CPPs are attached to the posterior medullary velum [14]. The free movement of the tumors within the ventricles due to the pedicular attachment allows for an acute gravity dependent intermittent obstruction of the ventricles giving rise to bobble head doll syndrome in some cases $[14,16]$. In our case also the tumor had a pedicular attachment arising from the choroid plexus in the temporal horn and the anterior choroidal artery forming its vascular supply.

\section{WHO grade I lesions}

CPPs belong to this group of lesions, and are histologically identified by a complex array of branching fibrovascular fronds covered by a monolayer of uniform cuboidal or low columnar epithelial cells. Nuclear atypia minimal and little mitotic activity (less than 2/10 high power field (HPF)) are seen. Stroma is infiltrated with foamy macrophages and may be pigmented by melanin. Areas of calcification, metaplastic bone or cartilage formation may be present. Epithelium may show oncocytic change, and may assume foci of ependymal differentiation producing GFAP-immunoreactive tapering cytoplasmic processes towards their fibrovascular cores. Exceptional cases exhibit a glandular growth pattern designated as acinar or tubular choroid plexus adenoma, confused with metastatic adenocarcinoma. Most peculiar is the CPP exhibiting overt neuronal differentiation, signaled by micronodular collection of synaptophysin rich matrix.

\section{WHO grade II lesions}

Atypical CPPs that fall short of over histological malignancy belong to this group. This lesion possesses area of multilayered epithelium of complex architecture, cytologic atypism, increased mitoses two or more mitoses per $10 \mathrm{HPF}$.

According to Gyure et al, lesions with conspicuous mitotic activity, necrosis, brain invasion and/or loss of architectural pattern but lacking the degree of anaplasia for diagnosis of carcinoma, are categorized as atypical CPPs.

\section{WHO grade III lesions}

Frankly malignant lesions, which are difficult to differentiate from metastatic adenocarcinoma, belong to this group. Grade
III lesions are de novo malignant lesions, very rare tumors present in infancy or childhood and are situated in a lateral ventricle. Microscopically, one sees nuclear pleomorphism, frequent mitoses, high nuclear to cytoplasmic ratio, increased cell density, loss of papillary architecture, necrosis and brain invasion. The mean Ki-67 (MIB-1) labeling index for CPP is 1.9\% and for choroid plexus carcinoma is $13.8 \%$.

\section{Immunohistochemistry}

Almost all CPPs express cytokeratin (CK7) and cytoplasmic vimentin. The majority $(80-90 \%)$ of them are labeled by antibody to transthyretin (TTR) and S-100 protein in the nuclei. Developmentally, the choroid plexus cells express GFAP, a glial marker, which persists in very few cells in adulthood. This is reflected in small number of tumor cells in $25-55 \%$ of papillomas. Immunolabeling for aquaporin 1, a water channel protein, is seen in normal choroid plexus and in CPPs. Altered p53 expression is seen in one-fourth of these papillomas.

On magnetic resonance (MR) images, CPPs are brilliantly enhancing intraventricular, lobulated masses, iso-intense on T1W and well defined, and hyperintense on T2W images [15, 16]. Flow voids are common which indicate flowing blood [14, 17]. To rule out the possibility of any seedling from these tumors, a follow-up with a contrast-enhanced MRI of spine may be required postoperatively [18]. CT scans reveal iso-dense or hyperdense lesions. MR spectroscopy shows higher levels of myo-inositol in these papillomas.

On ultrasonography through the anterior fontanelle, CPPs often appear as uniformly echogenic lobulated structures. Throughout the diastole, they continue to demonstrate a bidirectional flow and a ragged outline which is indicative of a blood flow in a bunch of vessels arranged in a chaotic fashion $[19,20]$. Both angiographic as well as cross-sectional imaging show choroidal artery enlargement [12].

Initially most attempts to resect these tumors were unfruitful thus pushing the mortality rate for these procedures to a very high level [21, 22]. Since 1902, when the first attempt was made to surgically remove a CPP, surgery has shown advancements in skills, articulations, techniques and instrumentations. The safe securing of the vascular pedicle of these tumors by its surgical ligation or by embolization on a preoperative setting has reduced the mortality rate for the attempts at surgical resection of these tumors to a ground touching low level in today's date $[15,23]$. This has led to an overall excellent prognosis for patients with CPPs and a 100\% recurrence free survival rate at 5 years in a certain large series $[12,15]$. There seems to be limited to no role of adjuvant therapy in these patients $[12,15]$. High quality MR angiography is very helpful in most cases [23]. Now with the availability of flexible catheters and innovations in catheter designs, the tortuous choroidal arteries seem no longer inaccessible and have made the embolization possible in a preoperative setting [12, 23, 24]. Another school of practice has been percutaneous stereotactic intratumoral embolization with the help of a sclerosing agent which helps in reducing the tumoral blood flow and making the surgical procedure much less tedious and risk free [12].

Milhorat et al [25] have shown that the rate of CSF forma- 
tion in a 5-year-old child who had undergone bilateral choroid plexectomy for communicating hydrocephalus during infancy was $0.35 \mathrm{~mL} / \mathrm{min}$, plus -0.02 standard deviation: well within normal limits. So it has been thoroughly and extensively documented that choroid plexectomy is not the answer for hydrocephalus. Consequently, the CSF secreted from extra choroidal sites accounts for the CSF increase though it continues to be secreted at a normal rate from the choroidal sites. So the hypersecretory type of hydrocephalus which results from a CPP may not necessarily be cured when the papilloma is resected.

There is secretion of high levels of protein by the papillary tumor which results in irreversible changes at the sites of CSF absorption. Consequently, it gives rise to raised CSF levels. A medium to high pressure shunt may always be needed to be inserted so as to render the postoperative course smooth and to diminish the possibility of progressive ventricular enlargement.

For these CPPs, complete resection is more frequently possible and is the most important prognostic factor. After gross resection of the papilloma, recurrences are rare and the functional outcome is excellent in $92 \%$ with a median survival of 75.5 months.

\section{Conclusion}

CPPs are rare entity in themselves and even rarer arising from the temporal horn as noted in this case. The management of these tumors is influenced by resectability, vascularity, presence of craniospinal spread and treatment of associated hydrocephalus. The ever increasing knowledge about these tumors makes the fact even stronger that a simple choroid plexectomy would not have sufficed in such cases and the placement of a VP shunt is equally important for a smooth postoperative recovery and diminishing the possibility of progressive ventricular enlargement.

\section{Conflicts of Interest}

None.

\section{Funding}

None.

\section{References}

1. Aguzzi A, Brandner S, Paulus W. Choroid plexus tumours. In: Kleihues P, Cavenee W, eds. Pathology and genetics of tumours of the nervous system. Lyon, France: IARC, 2000; 84-86.

2. Martin N, Pierot L, Sterkers O, Mompoint D, Nahum H. Primary choroid plexus papilloma of the cerebellopontine angle: MR imaging. Neuroradiology. 1990;31(6):541543.
3. Jackson A, Panizza BJ, Hughes D, Reid H. Primary choroid plexus papilloma of the cerebellopontine angle: magnetic resonance imaging, computed tomographic and angiographic appearances. Br J Radiol. 1992;65(777):754-757.

4. van Swieten JC, Thomeer RT, Vielvoye GJ, Bots GT. Choroid plexus papilloma in the posterior fossa. Surg Neurol. 1987;28(2):129-134.

5. Kimura M, Takayasu M, Suzuki Y, Negoro M, Nagasaka T, Nakashima N, Sugita K. Primary choroid plexus papilloma located in the suprasellar region: case report. Neurosurgery. 1992;31(3):563-566.

6. Garcia-Valtuille R, Abascal F, Garcia-Valtuille AI, Pinto JI, Cerezal L, Sanz F, Trigueros F, et al. Adult choroid plexus papilloma of the posterior fossa mimicking a hemangioblastoma. Case report. J Neurosurg. 2000;92(5):870-872.

7. Steven DA, McGinn GJ, McClarty BM. A choroid plexus papilloma arising from an incidental pineal cyst. AJNR Am J Neuroradiol. 1996;17(5):939-942.

8. Tasdemiroglu E, Awh MH, Walsh JW. MRI of cerebellopontine angle choroid plexus papilloma. Neuroradiology. 1996;38(1):38-40.

9. Nakano I, Kondo A, Iwasaki K. Choroid plexus papilloma in the posterior third ventricle: case report. Neurosurgery. 1997;40(6):1279-1282.

10. Sener RN. Choroid plexus papilloma of the temporal horn associated with transtentorial herniation. Comput Med Imaging Graph. 1998;22(3):263-264.

11. Phi JH, Chung CK, Lee YK, Chi JG, Kim HJ. Temporal lobe epilepsy caused by choroid plexus papilloma in the temporal horn. Clin Neuropathol. 2004;23(3):95-98.

12. Pencalet P, Sainte-Rose C, Lellouch-Tubiana A, Kalifa C, Brunelle F, Sgouros S, Meyer P, et al. Papillomas and carcinomas of the choroid plexus in children. J Neurosurg. 1998;88(3):521-528.

13. Ellenbogen RG, Winston KR, Kupsky WJ. Tumors of the choroid plexus in children. Neurosurgery. 1989;25(3):327335.

14. Coates TL, Hinshaw DB, Jr., Peckman N, Thompson JR, Hasso AN, Holshouser BA, Knierim DS. Pediatric choroid plexus neoplasms: $\mathrm{MR}, \mathrm{CT}$, and pathologic correlation. Radiology. 1989;173(1):81-88.

15. McGirr SJ, Ebersold MJ, Scheithauer BW, Quast LM, Shaw EG. Choroid plexus papillomas: long-term followup results in a surgically treated series. J Neurosurg. 1988;69(6):843-849.

16. Pollack IF, Schor NF, Martinez AJ, Towbin R. Bobblehead doll syndrome and drop attacks in a child with a cystic choroid plexus papilloma of the third ventricle. Case report. J Neurosurg. 1995;83(4):729-732.

17. Reimund EL, Sitton JE, Harkin JC. Pigmented choroid plexus papilloma. Arch Pathol Lab Med. 1990;114(8):902905.

18. Vazquez E, Ball WS, Jr., Prenger EC, Castellote A, Crone KR. Magnetic resonance imaging of fourth ventricular choroid plexus neoplasms in childhood. A report of two cases. Pediatr Neurosurg. 1991;17(1):48-52.

19. Cappe IP, Lam AH. Ultrasound in the diagnosis of choroid plexus papilloma. J Clin Ultrasound. 1985;13(2):121- 
123.

20. Chow PP, Horgan JG, Burns PN, Weltin G, Taylor KJ. Choroid plexus papilloma: detection by real-time and Doppler sonography. AJNR Am J Neuroradiol. 1986;7(1):168-170.

21. Kahn EA, Luros JT. Hydrocephalus from overproduction of cerebrospinal fluid, and experiences with other parillomas of the choroid plexus. J Neurosurg. 1952;9(1):59-67.

22. Bohm E, Strang R. Choroid plexus papillomas. J Neurosurg. 1961;18:493-500.
23. Nagib MG, O'Fallon MT. Lateral ventricle choroid plexus papilloma in childhood: management and complications. Surg Neurol. 2000;54(5):366-372.

24. Do HM, Marx WF, Khanam H, Jensen ME. Choroid plexus papilloma of the third ventricle: angiography, preoperative embolization, and histology. Neuroradiology. 2001;43(6):503-506.

25. Milhorat TH, Hammock MK, Davis DA, Fenstermacher JD. Choroid plexus papilloma. I. Proof of cerebrospinal fluid overproduction. Childs Brain. 1976;2(5):273-289. 